\title{
Higher order accuracy in the gap-tooth scheme for large-scale dynamics using microscopic simulators
}

\author{
A. J. Roberts* $\quad$ I. G. Kevrekidis ${ }^{\dagger}$ \\ (Received 15 October 2004, revised 29 April 2005)
}

\begin{abstract}
We are developing a framework for multiscale computation which enables models at a "microscopic" level of description, for example Lattice Boltzmann, Monte-Carlo or Molecular Dynamics simulators, to perform modelling tasks at the "macroscopic" length scales of interest. The plan is to use the microscopic rules restricted to small patches of the domain, the "teeth", followed by interpolation to estimate macroscopic fields in the "gaps". The challenge begun here is to find general boundary conditions for the patches of microscopic simulators that appropriately connect the widely separated "teeth" to
\end{abstract}

*Dept. Maths \& Computing, University of Southern Queensland, Toowoomba, Australia. mailto: aroberts@usq.edu.au

${ }^{\dagger}$ Program in Applied and Computational Mathematics, Princeton University, Princeton, NJ 08544, USA. mailto:yannis@Princeton.edu

See http://anziamj.austms.org.au/V46/CTAC2004/Robe for this article, (C) Austral. Mathematical Soc. 2005. Published July 20, 2005. ISSN 1446-8735 
achieve high order accuracy over the macroscale. Here we start exploring the issues in the simplest case when the microscopic simulator is the quintessential example of a partial differential equation. In this case analytic solutions provide comparisons. We argue that classic high-order interpolation provides patch boundary conditions which achieve arbitrarily high-order consistency in the gap-tooth scheme, and with care are numerically stable. The high-order consistency is demonstrated on a class of linear partial differential equations in two ways: firstly, using the dynamical systems approach of holistic discretisation; and secondly, through the eigenvalues of selected numerical problems. When applied to patches of microscopic simulations these patch boundary conditions should achieve efficient macroscale simulation.

\section{Contents}

1 Introduction

C638

2 Couple the patches

C644

3 Achieve high order consistency

C646

4 The diffusive model is numerically stable

C650

5 Conclusion

C653

References

C654

\section{Introduction}

This research is part of a wide ranging project to create a set of computational super-structures (libraries) to wrap around whatever the best mi- 
croscopic level computer model a scientist would come up with for her/his system [10, 9, e.g.] - be it a Monte-Carlo description of a chemical reaction or an individual-based model in ecology or epidemiology. A persistent feature of complex systems is the emergence of macroscopic, coherent behavior from the interactions of microscopic agents - molecules, cells, individuals in a population-between themselves and with their environment. The implication is that macroscopic rules (description of behaviour at a large scale) can somehow be deduced from microscopic ones (description of behavior at a finer level). Crucially, we address how information is transformed between scales. For some problems (like Newtonian fluid mechanics) the successful macroscopic description (the Navier-Stokes equations) predated its microscopic derivation from kinetic theory. However, in many current problems, ranging from ecology [4, e.g.] to materials science [14, 8, e.g.], and from chemistry to engineering [24, e.g.], the physics are known at the microscopic/individual level, but the closures required to translate them to a high-level macroscopic description are simply not available analytically [22], except in some simple circumstances [1]. Further, three invited speakers at the International Congress on Industrial and Applied Mathematics in 2003 emphasised the importance of, and discussed, the sort of multiscale modelling that this project addresses: Thomas Hou, Multiscale modelling and computation of incompressible flow; Michael Ortiz, Variational problems in mechanics and the link between microstructure and macroscopic behaviour; and David L. Donoho, Geometric multiscale analysis and its applications.

Dolbow, Khaleel \& Mitchell [5] report to the us Department of Energy that

Science and technology are on the brink of a revolution. Physical processes at exceedingly small scales of time and space are becoming increasingly well understood. Technologies for engineering systems at the micro and nano scales are rapidly emerging. Yet in general we have no means of translating fundamental, detailed scientific knowledge at small scales into its effects on the 


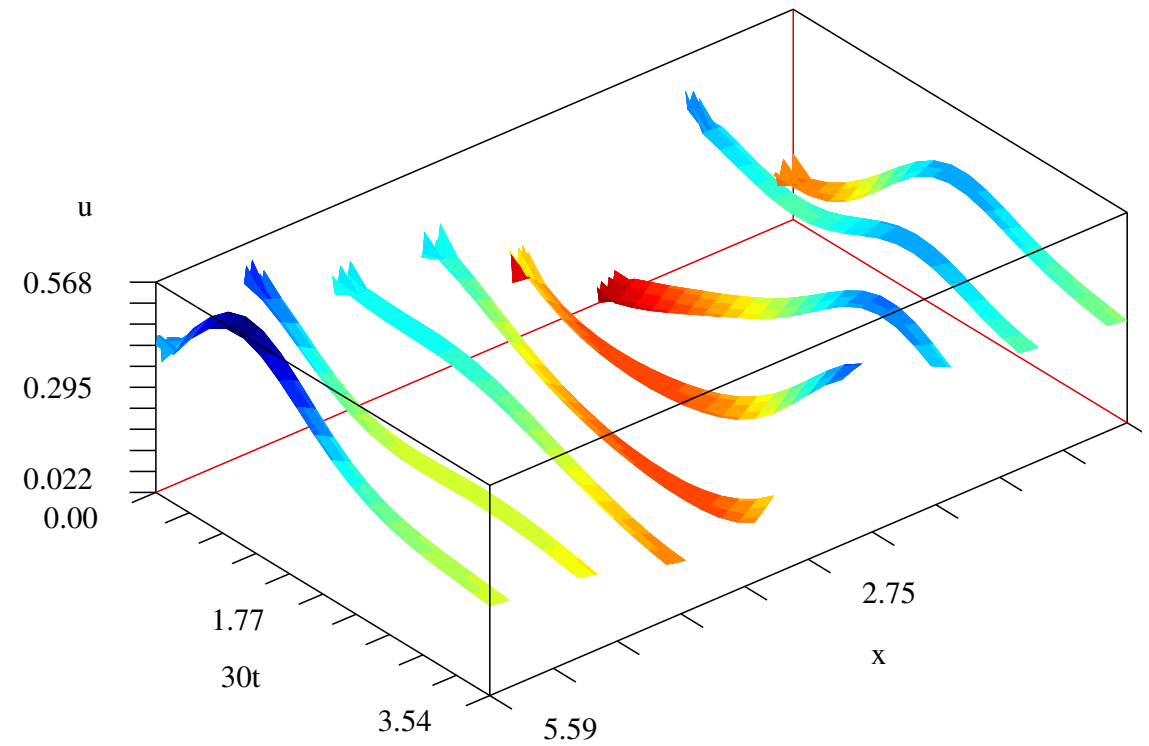

Figure 1: Gap-tooth solution of Burgers' equation (1) on $[0,2 \pi]$ through microsimulation on 8 teeth, each of small width $\pi / 20$; the teeth are coupled by special patch boundary conditions.

macroscopic world in which we live. Without the capability to "bridge the scales," important scientific and engineering problems will remain out of reach.

Subsequently the Department of Energy called for applications for research grants to be allocated "up to [US]\$5.8 million in this ... Fiscal Year 2005". We aim to contribute to multiscale modelling by circumventing the lack of analytic closures by our novel computational methods; just one example in our project is the macroscale modelling of diffusion in random media [23]. Our initial work here indicates how the gap-tooth scheme [7, 22, e.g.] may connect microscale patches to achieve higher order accuracy in the macroscale. 
As a preliminary illustration of the gap-tooth scheme [7, 21, 22], consider simulating the diffusion and nonlinear advection of the viscous Burgers' equation

$$
\frac{\partial u}{\partial t}+100 u \frac{\partial u}{\partial x}=\frac{\partial^{2} u}{\partial x^{2}} .
$$

Suppose our aim is to simulate the evolution of fields $u(x, t)$ periodic in $x$ on the macroscopic length scale $2 \pi$. See in Figure 1 the continuous time evolution on $m=8$ grid "points" in space with macroscopic spacing $H=$ $\pi / 4$. However, each "point" is actually a microscopic patch of width $h=$ $\pi / 20$. Further, the only knowledge that the macroscopic evolution has of Burgers' PDE (1) is through the detailed simulation of the PDE within each patch [9]; here we obtain this local detailed simulation via a discretisation of (1) on a microscopic spatial grid of $n=11$ points within each patch, $\Delta x=0.0175$, and on a microscopic time step of $\Delta t \approx 10^{-4}$. This fine scale discretisation of Burgers' PDE (1) represents a finely detailed model or particle simulation that is too expensive to use over the entire macroscopic domain $[9,11]$. Recall that the methodology is to evaluate automatically ("on demand") the macroscopic modelling closures which all too often are not available explicitly [4, 22, e.g.]. Our task here is to begin to show how such microscopic simulations in small patches of space may be coupled by patch boundary conditions derived in Section 2 to ensure high order accuracy over the macroscopic domain. Here we use a well understood PDE only so we can analyse explicitly all details of the transformation from microscale model, the PDE, to macroscale discretisation.

The example of Figures 1 and 2 shows us that there are two time scales in the simulation. Rapidly, the initial internal structure within each tooth (black curves in Figure 2) smooths by diffusion on the microscopic timescale to a local quasi-equilibrium (blue curves). Then, over longer times the inter-patch coupling exchanges information between the teeth to guide how the local quasi-equilibria evolve over macroscopic times. See the dynamics in Figure 2: the broad hump initially centred around $x \approx 4$ is nonlinearly advected to the right to wrap around to about $x \approx 1$ at the end (red curves); 


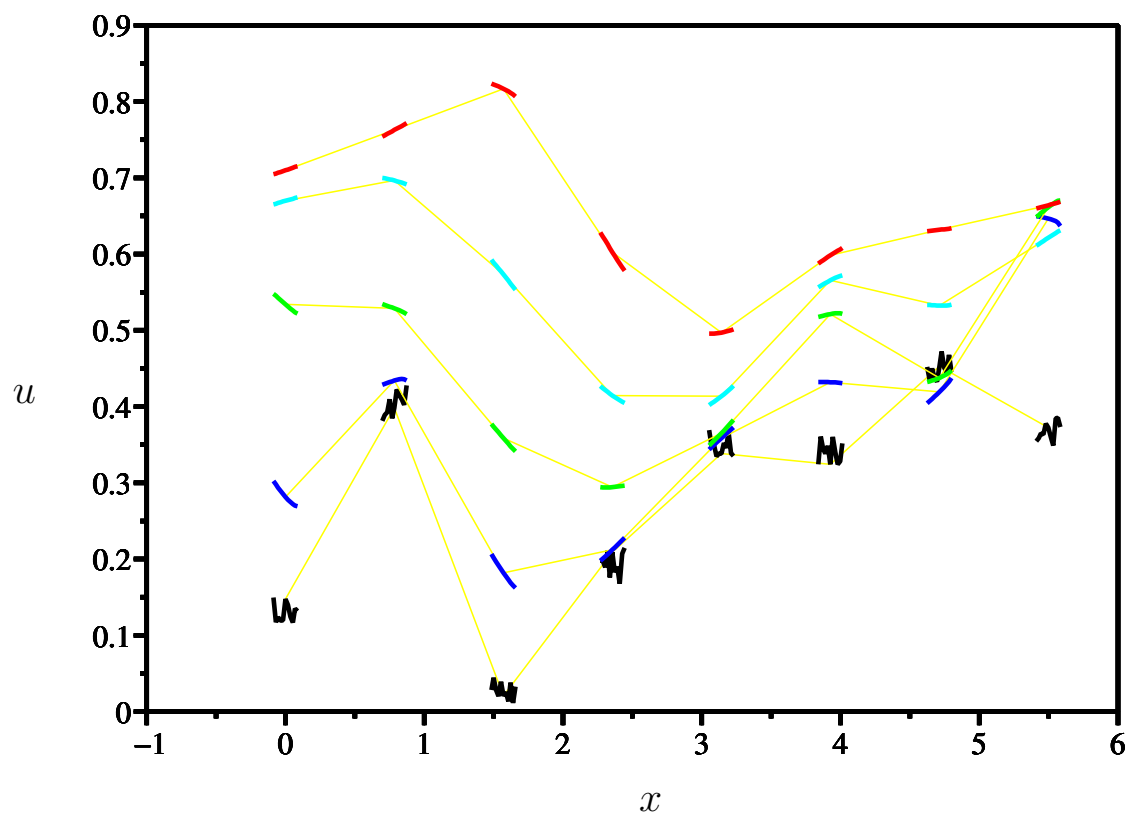

Figure 2: Gap-tooth solution of Burgers' equation (1) on $[0,2 \pi]$ on 8 teeth each of small width $\pi / 20$ and coupled by special patch boundary conditions. Solutions $u(x, t)+4 t$ are plotted at five times $t=0: 0.025: 0.1$ in different colours and connected by yellow lines and with the vertical displacement of $4 t$ to help distinguish the plots.

whereas the short hump initially at $x \approx 1$ is dissipated quickly against the slow moving region at $x \approx 2$. The rapid smoothing of internal structure includes the elimination of any boundary layers that might occur near the edges of each patch - the forcing of each patch by the macroscopic grid values $U_{j}$ varies on a time scale much longer than the time scale of decay internal to each patch, later seen explicitly in Tables 1, 2 and 3, and consequently the structure internal to each patch is smooth.

Because of the two time scales, we plan future research to implement 
"coarse grained" integration [6] which uses just short bursts of microscopic integration to then extrapolate over a macroscopic time step. The result will then be a scheme where the microscopic simulations are only needed for relatively small patches in space-time. However, here we concentrate on only the issue of the macroscopic coupling of small patches across space.

The method of "holistic discretisation", developed by Roberts \& Mackenzie $[17,18,19,13]$, creates discretisations on a macroscopic grid using systematically obtained analytic approximations for the subgrid field. The analytic solutions of Section 3 using this method are analogous to the microscopic system simulators in the gap-tooth scheme: they both provide microscopic solutions which are macroscopically coupled to neighbouring elements. This dynamical systems approach adapts the patch boundary conditions to support the modelling by centre manifold theory [18]. Then the equivalent PDE of the macroscopic dynamic model is found, in Section 3, to confirm high order consistency for a wide class of linear PDEs. Although the theoretical support for our analytic approach here is apparently based upon dissipation being a dominant mechanism, the approach works remarkably well for large advection (large in comparison with diffusive dissipation). Roberts [18, §3] found that when subgrid scale structures are solved for finite advection, in a manner more closely analogous to the information provided by microscale simulators, then the macroscale discretisation transforms smoothly between high order accurate centred difference schemes with small advection to lower order but robust upwind discretisations with very large advection.

Lastly, in Section 4, we consider a numerical time integrator for the diffusion equation on patches. The eigenvalues of the integrator again confirm the high order accuracy of the proposed patch boundary conditions. 


\section{Couple the patches}

In this section we develop a coupling of the internal dynamics of patches with their neighbours to achieve high order consistency. We construct a boundary condition for the flux on the edge of the microscopic patches that is a natural interpolation of the surrounding macroscopic field. The number of required boundary conditions will depend upon the microscopic simulator [12, e.g.], and possibilities other than the flux remain to be explored. But here we know that boundary conditions on the flux should give rise to well-posed diffusion-like dynamics.

We introduce the notation in which we typically use capital letters for macroscopic quantities and lower case letters for microscopic quantities. Thus let each of $m$ patches be centred on equi-spaced grid points $x=X_{j}=j H$ seen in Figures 1 and 2. Let each patch be of width $h$. Then the edge of a patch is a distance $h / 2$ from its grid point, a fraction $r=h /(2 H)$ to the neighbouring grid point: when $r=\frac{1}{2}$ the neighbouring patches meet and there would be no gap, as in holistic discretisation [17]. Here we expect the fraction $r$ to be small so that the patches are a relatively small part of the physical domain. For example, $r=1 / 10$ in Figures 1 and 2. Now let $v_{j}(x, t)$ be the microscopic field in the $j$ th patch.

We use the following identities for discrete operators [15] on a step size of the macroscopic grid and are careful whether we are using as a step of $H$ in $x$ or a step of 1 in $j$. In terms of the shift operator, $E v(x, t)=v(x+H, t)$ or equivalently $E U_{j}=U_{j+1}$ :

$$
\text { centred mean } \quad \mu=\frac{1}{2}\left(E^{1 / 2}+E^{-1 / 2}\right),
$$

centred difference

$\delta=E^{1 / 2}-E^{-1 / 2}$,

translate/shift $\quad E=1+\mu \delta+\frac{1}{2} \delta^{2}$,

derivative in $x$

$$
H \partial_{x}=2 \sinh ^{-1} \frac{1}{2} \delta,
$$

an identity

$$
\mu^{2}=1+\frac{1}{4} \delta^{2} \text {. }
$$


Exponents behave naturally, for examples $E^{2} U_{j}=E\left(E U_{j}\right)=U_{j+2}$, whereas $E^{-1 / 2} v(x, t)=v(x-H / 2, t)$, and so on. Consequently, obtain the derivative of the microscopic field on the edge of a patch, $H \frac{\partial v_{j}}{\partial x}$ at $\left(X_{j} \pm r H, t\right)$, from $v_{j}$ through applying the operator

$$
\begin{aligned}
E^{ \pm r} H \partial_{x} & =\left(1+\mu \delta+\frac{1}{2} \delta^{2}\right)^{ \pm r} 2 \sinh ^{-1} \frac{1}{2} \delta \quad \text { by (4) and (5) } \\
& =\left[1 \pm r \mu \delta+\mathcal{O}\left(\delta^{2}\right)\right]\left[\delta+\mathcal{O}\left(\delta^{3}\right)\right] \\
& =\delta \pm r \mu \delta^{2}+\mathcal{O}\left(\delta^{3}\right) \\
& =\mu \delta \pm r \delta^{2}+\mathcal{O}\left(\delta^{3}\right) \quad \text { by }(6)
\end{aligned}
$$

This last operator just involves evaluation at the grid points $X_{j}$ and hence is evaluated from the macroscopic grid values $U_{j}$. This provides the same approximation for the microscopic gradient as obtained by quadratic interpolation through the neighbouring macroscopic grid values [7, e.g.]. We proceed to modify such a patch boundary condition in order to obtain higher order consistency with the surrounding macroscopic variations.

For arbitrary order consistency, as the macroscopic grid size $H \rightarrow 0$ or as the macroscopic gradients become small, repeat the previous analysis but retain more terms. Using (6) to replace $\mu^{2}$ terms:

$$
\begin{aligned}
E^{ \pm r} H \partial_{x}= & \left(1+\mu \delta+\frac{1}{2} \delta^{2}\right)^{ \pm r} 2 \sinh ^{-1} \frac{1}{2} \delta \\
= & \frac{\mu}{\sqrt{1+\frac{1}{4} \delta^{2}}}\left(1+\mu \delta+\frac{1}{2} \delta^{2}\right)^{ \pm r} 2 \sinh ^{-1} \frac{1}{2} \delta \\
= & \mu \delta \pm r \delta^{2}-\left(\frac{1}{6}-\frac{1}{2} r^{2}\right) \mu \delta^{3} \mp r\left(\frac{1}{12}-\frac{1}{6} r^{2}\right) \delta^{4} \\
& +\left(\frac{1}{30}-\frac{1}{8} r^{2}+\frac{1}{24} r^{4}\right) \mu \delta^{5} \pm r\left(\frac{1}{90}-\frac{1}{36} r^{2}+\frac{1}{120} r^{4}\right) \delta^{6} \\
& -\left(\frac{1}{140}-\frac{7}{240} r^{2}+\frac{1}{72} r^{4}-\frac{1}{720} r^{6}\right) \mu \delta^{7} \\
& \mp r\left(\frac{1}{560}-\frac{7}{1440} r^{2}+\frac{1}{480} r^{4}-\frac{1}{5040} r^{6}\right) \delta^{8}+\mathcal{O}\left(\delta^{9}\right) .
\end{aligned}
$$

Numerical eigenanalysis of the diffusion equation (16) reported in Section 4 confirms the high order accuracy and stability of the resultant integration scheme with patch boundary conditions from the above operator. 


\section{Achieve high order consistency}

Here we demonstrate analytically that appropriate patch boundary conditions achieve high order consistency for a wide class of PDEs. Recall that these PDEs represent microscopic simulators: hence it is crucial that our patch boundary conditions (PtBCs) are accurate for general PDEs so that they will also be accurate for general microscopic dynamics whose PDEs we generally will not know. Consider the linear PDE

$$
\frac{\partial u}{\partial t}=\frac{\partial^{2} u}{\partial x^{2}}-c \frac{\partial u}{\partial x}-b \frac{\partial^{3} u}{\partial x^{3}}-a \frac{\partial^{4} u}{\partial x^{4}},
$$

for some constants $a, b$ and $c$-we have chosen time and space scales so that the coefficient of the diffusion term is 1 . Specially crafted boundary conditions on small patches ensures macroscopic consistency.

Following the dynamical systems approach of holistic discretisation [17, 18] we introduce the parameter $\gamma$ to control the coupling between patches: when $\gamma=0$ the patches are uncoupled to provide a base for us to apply centre manifold theory; but when we subsequently set $\gamma=1$ we recover a dynamical model for the original PDE. Modify the operator (8) to invoke the PtBC that on $x=X_{j}$ (noting that the $E^{ \pm r}$ implies the left-hand side is evaluated on the edge of the patch at $\left.x=X_{j} \pm r H\right)$ :

$$
\begin{aligned}
E^{ \pm r} H \partial_{x} v_{j}=\{ & \left\{\left[\mu \delta \pm r \delta^{2}\right]+\gamma^{2}\left[-\left(\frac{1}{6}-\frac{1}{2} r^{2}\right) \mu \delta^{3} \mp r\left(\frac{1}{12}-\frac{1}{6} r^{2}\right) \delta^{4}\right]\right. \\
& +\gamma^{3}\left[+\left(\frac{1}{30}-\frac{1}{8} r^{2}+\frac{1}{24} r^{4}\right) \mu \delta^{5} \pm r\left(\frac{1}{90}-\frac{1}{36} r^{2}+\frac{1}{120} r^{4}\right) \delta^{6}\right] \\
+ & \gamma^{4}\left[-\left(\frac{1}{140}-\frac{7}{240} r^{2}+\frac{1}{72} r^{4}-\frac{1}{720} r^{6}\right) \mu \delta^{7}\right. \\
& \left.\left.\mp r\left(\frac{1}{560}-\frac{7}{1440} r^{2}+\frac{1}{480} r^{4}-\frac{1}{5040} r^{6}\right) \delta^{8}\right]\right\} U_{j} .
\end{aligned}
$$

Neglecting the $\mathcal{O}\left(\gamma^{4}\right)$ terms, this PtBC was implemented for the simulation in Figures 1 and 2. See in these PtBCs that when $\gamma=0$ the small patches are decoupled and the resulting insulating boundary conditions, $E^{ \pm r} \partial_{x} v_{j}=0$, cause the dissipative dynamics of (9) in each patch to decay exponentially quickly to some constant field in each patch, namely $v_{j}(x, t) \rightarrow U_{j}$ for each of 
the $m$ patches. ${ }^{1}$ For non-zero coupling parameter $\gamma$ the subgrid scale patch field is no longer constant, and each patch grid value $U_{j}$ evolves because of the coupling with its neighbours. We construct a series solution of the PDE (9) in the coupling parameter $\gamma$ : the first order expression for the microscopic subgrid scale field is straightforward, namely

$$
v_{j}=U_{j}+\gamma\left(\xi \mu \delta+\frac{1}{2} \xi^{2} \delta^{2}\right) U_{j}+c \gamma H\left(-\frac{1}{2} r^{2} \xi+\frac{1}{6} \xi^{3}\right) \delta^{2} U_{j}+\mathcal{O}\left(\gamma^{2}, a^{2}+b^{2}+c^{2}\right),
$$

where the microscopic variable $\xi=\left(x-X_{j}\right) / H$ ranges over $|\xi|<r$; accompanying these subgrid fields the grid values $U_{j}$ evolve according to standard second order discretisation (upon putting $\gamma=1$ )

$$
\dot{U}_{j}=\gamma\left(\frac{1}{H^{2}} \delta^{2} U_{j}-\frac{c}{H} \mu \delta U_{j}\right)+\mathcal{O}\left(\gamma^{2}, a^{2}+b^{2}+c^{2}\right) .
$$

See that the powers of the coupling parameter $\gamma$ in the PtBC (10) are chosen so that discarding terms of $\mathcal{O}\left(\gamma^{p}\right)$ results in a discrete model, such as (12), which is of width $2 p-1$ in the grid values $U_{j}$; for example, the above model only involves $U_{j}$ and $U_{j \pm 1}$. Centre manifold theory [2,3, e.g.] asserts that for small enough $\gamma$ all neighbouring solutions are exponentially quickly attracted to the resultant model which faithfully describes the dynamics of the system. Although no proof is yet available, we anticipate that the case of interest, when $\gamma=1$, is small enough for this novel theoretical support to still hold.

In the interim we demonstrate high order consistency. We obtain models that resolve more detail of the subgrid microscopic dynamics and its interaction with neighbouring patches by determining higher order terms in the

${ }^{1}$ Why are we only using one pair of boundary conditions for the apparently fourth order PDE (9)? One answer is that the PDE may be viewed as an equivalent PDE for a microscopic simulator that only requires one pair of boundary conditions. For example, if the microscopic simulator is simply a fine scale discretisation of a PDE, such as $u_{t}=-c(\delta / h) u+\left(\delta^{2} / h^{2}\right) u$, then only one pair of boundary conditions are needed for the simulator, but it has a high order equivalent PDE such as (9). Another answer is that there is no physical boundary at the edge of a patch and so we only need resolve smooth subgrid fields. For smooth solutions we need only treat the higher order terms as perturbations; see that our error terms are expressed as $\mathcal{O}\left(a^{2}+b^{2}+c^{2}\right)$ for this reason. 
coupling parameter $\gamma$. Iteration [16] straightforwardly generates higher order approximations using computer algebra [20]. For example, discarding terms $\mathcal{O}\left(\gamma^{3}\right)$ the subgrid field in each patch is modified from (11) to

$$
\begin{aligned}
v_{j}= & \left\{1+\gamma\left[\xi \mu \delta+\frac{1}{2} \xi^{2} \delta^{2}\right]+\gamma^{2}\left[\frac{1}{6}\left(\xi^{3}-\xi\right) \mu \delta^{3}+\frac{1}{24}\left(\xi^{2}-\xi^{4}\right) \delta^{4}\right]\right. \\
& +c H\left[\left(\gamma-\gamma^{2}\right) \frac{1}{6} \xi^{3} \delta^{2}+\gamma^{2}\left(\frac{1}{60} \xi^{5}-\frac{1}{18} \xi^{3}\right) \delta^{4}\right]+\frac{b}{H} \gamma^{2} \frac{1}{6} \xi^{3} \delta^{4} \\
& +r^{2}\left[-c H\left(\gamma-\gamma^{2}\right) \frac{1}{2} \xi \delta^{2}-c H \gamma^{2}\left(\frac{1}{12} \xi^{3}-\frac{1}{6} \xi\right) \delta^{4}-\frac{b}{H} \gamma^{2} \frac{1}{2} \xi \delta^{4}\right] \\
& \left.+r^{4} c H \frac{1}{6} \xi \delta^{4}\right\} U_{j}+\mathcal{O}\left(\gamma^{3}, a^{2}+b^{2}+c^{2}\right) .
\end{aligned}
$$

The first line in (13) contains the leading few terms in a universal subgrid structure for symmetric operators. However, odd operators, such as the advection $c \frac{\partial u}{\partial x}$ and the dispersion $b \frac{\partial^{3} u}{\partial x^{3}}$, generate nontrivial subgrid structures in each patch, such as those in the second line of (13), which reflect subgrid scale interaction of processes. The third and fourth line of the approximate field (13) depend upon the patch size $r=h /(2 H)$. But physically the subgrid scale field in each patch should be independent of the patch size $r$. Although there is some dependence in these approximations, higher orders in the coupling parameter $\gamma$ remove it. For example, at the beginning of the third line in (13) see the term $-c H\left(\gamma-\gamma^{2}\right) \frac{1}{2} \xi \delta^{2}$ disappears when we set $\gamma=1$ for the physically relevant approximation. Similarly, computing the next order terms in coupling parameter $\gamma$ generates terms, in $\gamma^{3}$, which cancel the $r$ dependent terms in the third and fourth line of the subgrid field (13). Thus higher order models push any undesirable $r$ dependence to higher orders, thereby usefully predicting a subgrid field largely independent of the patch size $r$.

Simultaneously with the derivation of the subgrid field (13) we determine the corresponding evolution of the macroscopic grid values $U_{j}$ for the PDE (9). Computing to higher order in the coupling parameter $\gamma$ produces refinements 
to the basic discretisation (12); for example, here we discard terms $\mathcal{O}\left(\gamma^{4}\right)$ to determine

$$
\begin{aligned}
\dot{U}_{j}= & \frac{1}{H^{2}}\left(\gamma \delta^{2}-\frac{1}{12} \gamma^{2} \delta^{4}+\frac{1}{90} \gamma^{3} \delta^{6}\right) U_{j}-\frac{c}{H}\left(\gamma \mu \delta-\frac{1}{6} \gamma^{2} \mu \delta^{3}+\frac{1}{30} \gamma^{3} \mu \delta^{5}\right) U_{j} \\
& -\frac{b}{H^{3}}\left(\gamma^{2} \mu \delta^{3}-\frac{1}{4} \gamma^{3} \mu \delta^{5}\right) U_{j}-\frac{a}{H^{4}}\left(\gamma^{2} \delta^{4}-\frac{1}{6} \gamma^{3} \delta^{6}\right) U_{j} \\
& +\mathcal{O}\left(\gamma^{4}, a^{2}+b^{2}+c^{2}\right) .
\end{aligned}
$$

Set $\gamma=1$ to recover a model for the PDE (9) supported by centre manifold theory. Note how truncating the expansion to different powers of coupling parameter $\gamma$ changes the width in $U_{j}$ of the discrete model. With the patch boundary conditions (10) the model is independent of the patch size $r$.

As well as the novel dynamical systems support of exponentially quick attractiveness and long term fidelity at finite grid size $H$, as mentioned earlier, another way to assess the model's relevance is to compare the original PDE with the equivalent PDE obtained from model (14) in the limit as the macroscopic spacing $H \rightarrow 0$. From (14), straightforward algebra, see our REDUCE code [20], deduces the equivalent PDE

$$
\begin{aligned}
& \frac{\partial U}{\partial t}=\gamma \frac{\partial^{2} U}{\partial x^{2}}-\gamma c \frac{\partial U}{\partial x}-\gamma^{2} b \frac{\partial^{3} U}{\partial x^{3}}-\gamma^{2} a \frac{\partial^{4} U}{\partial x^{4}} \\
& +H^{2}\left[\left(\gamma-\gamma^{2}\right)\left(\frac{1}{12} \frac{\partial^{4} U}{\partial x^{4}}-\frac{1}{6} c \frac{\partial^{3} U}{\partial x^{3}}\right)-\left(\gamma^{2}-\gamma^{3}\right)\left(\frac{1}{4} b \frac{\partial^{5} U}{\partial x^{5}}+\frac{1}{6} a \frac{\partial^{6} U}{\partial x^{6}}\right)\right] \\
& +H^{4}\left[\left(\frac{1}{360} \gamma-\frac{1}{72} \gamma^{2}+\frac{1}{90} \gamma^{3}\right)\left(\frac{\partial^{6} U}{\partial x^{6}}-3 c \frac{\partial^{5} U}{\partial x^{5}}\right)\right. \\
& \left.\quad-\left(\frac{1}{80} \gamma^{2}-\frac{1}{24} \gamma^{3}\right)\left(b \frac{\partial^{7} U}{\partial x^{7}}+2 a \frac{\partial^{8} U}{\partial x^{8}}\right)\right]+\mathcal{O}\left(H^{6}, \gamma^{4}, a^{2}+b^{2}+c^{2}\right) .
\end{aligned}
$$

When the coupling parameter $\gamma=1$ the second and third lines in the equivalent PDE (15) disappear and consequently the diffusion and advection is modelled with errors of $\mathcal{O}\left(H^{6}\right)$, whereas the dispersion and the fourth-order dissipation is modelled with errors $\mathcal{O}\left(H^{4}\right)$. Should you truncate the discretisation (14) to lower orders in coupling parameter $\gamma$, there is less cancellation 
in the equivalent PDE and the errors are consequently larger. Conversely, the errors move to progressively higher orders as more terms in the coupling parameter $\gamma$ are retained in the centre manifold discretisation (14). Our patch boundary conditions (10) create excellent discretisations for quite general PDEs and hence should perform well for other microscale simulators.

\section{The diffusive model is numerically stable}

Although the PtBCs (10) form consistent models we need to confirm they are numerically stable. Indeed many other forms of PtBCs were tried before finding one that was both consistent and numerically stable. In this section we explore the gap-tooth simulations of the simple diffusion equation

$$
\frac{\partial u}{\partial t}=\frac{\partial^{2} u}{\partial x^{2}}, \quad \text { and } 2 \pi \text {-periodic in } x .
$$

Imagine we only have access to the dynamics through a microscopic simulator of the diffusion (16), here coded by a fine discretisation on $n$ grid points in a patch of microscopic size $h=r H$ and with some microscopic time step, typically $\Delta t=10^{-6}-10^{-4}$.

Firstly we implement the PtBC that on the edge of each patch the fine discretisation has boundary condition

$$
\left[\mu \delta \pm r \delta^{2}-\left(\frac{1}{6}-\frac{1}{2} r^{2}\right) \mu \delta^{3} \mp r\left(\frac{1}{12}-\frac{1}{6} r^{2}\right) \delta^{4}\right] U_{j}=H \partial_{x} v_{j} \quad \text { at } x=X_{j} \pm r H .
$$

Obtain this from the first few terms of (8) or equivalently from PtBC (10) by discarding $\mathcal{O}\left(\gamma^{3}\right)$ terms. Consider how this PtBC would be implemented in a microscopic particle simulation. Crucially it does not refer to individual particles but to the local estimate of the continuum gradient. Certainly, insulating boundary conditions may be implemented simply by reflecting outgoing particles, but in general more subtle treatments of the microscopic 
TABLE 1: Growth rates $\lambda$ of perturbations from steady state $u=0$ : for diffusion (16) with $m$ patches; with gap to patch ratio $r=0.1 ; n=11$ points in the microscale grid; and with the fourth order PtBC (17).

\begin{tabular}{|r|lccl|c|}
\hline$m$ & \multicolumn{1}{|c}{1} & 2,3 & 4,5 & 6,7 & $m+1: 2 m$ \\
\hline 4 & $2 \times 10^{-12}$ & -0.946817 & -2.170942 & $\mathrm{n} / \mathrm{a}$ & -99.79 \\
8 & $5 \times 10^{-12}$ & -0.996139 & -3.787268 & -7.132829 & -399.1 \\
16 & $2 \times 10^{-10}$ & -0.999758 & -3.984556 & -8.834269 & -1596. \\
32 & $-2 \times 10^{-10}$ & -0.999987 & -3.999031 & -8.988851 & -6386. \\
\hline
\end{tabular}

system near the boundary are needed depending upon the particle dynamics and the nature of the boundary condition. For example, specified value PtBCs will involve actions depending upon the local averages estimating the local field value. Here we are supposing that the microscopic simulator naturally implements boundary conditions of specified gradient, and so we discuss PtBCs in the form (17). More general forms of PtBCs will be explored in future research. One aim in our project is to provide analytic support for a wide range of microsimulator boundary conditions depending upon what is natural for the available particle simulator.

For the $j$ th patch this PtBC involves macroscopic grid values $U_{j-2}, \ldots, U_{j+2}$ only. Then systematically perturbing each and every microscopic value from zero, there are $m n$ such microscopic values, we numerically determined the map of one microscopic time step. ${ }^{2}$ Transform the eigenvalues $\mu$ of this map to growth rates $\lambda=\log (\mu) / \Delta t$. The $m n$ growth rates fall into $n$ groups of $m$ modes. Each group corresponds to a microscopic internal mode of the dynamics, roughly $\exp \left(\lambda_{\ell} t\right) \cos \left[\ell \pi\left(x-X_{j}+h / 2\right) / h\right]$ for growth rate $\lambda_{\ell} \approx-\ell^{2} \pi^{2} / h^{2}$ for $\ell=0,1, \ldots, n-1$. For $\ell \geq 1$ these are the rapidly decaying microscopic modes internal to each patch seen in the initial instants of the simulations of Figures 1 and 2. The other group of $m$ modes,

\footnotetext{
${ }^{2}$ In general, the dominant eigenvalues of the time-stepper map may be obtained via a matrix-free Krylov subspace iteration [21]. Thus for particle simulations we do not necessarily need access to all the fine details of the microscale.
} 
TABLE 2: Growth rates of perturbations from steady state $u=0$ as for Table 1 but fewer points in the fine grid, namely $n=7$.

\begin{tabular}{|r|llll|c|}
\hline$m$ & \multicolumn{1}{|c}{2,3} & 4,5 & 6,7 & $m+1: 2 m$ \\
\hline 4 & $8 \times 10^{-13}$ & -0.947206 & -2.173003 & n/a & -99.30 \\
8 & $-8 \times 10^{-12}$ & -0.996246 & -3.788826 & -7.138379 & -397.2 \\
16 & $-1 \times 10^{-11}$ & -0.999785 & -3.984985 & -8.836383 & -1588. \\
32 & $8 \times 10^{-11}$ & -0.999994 & -3.999139 & -8.989397 & -6355. \\
\hline
\end{tabular}

$\ell=0$, with small growth rates, correspond to the relatively slowly evolving macroscopic modes of interest that arise through the coupling between patches of the microscopic dynamics. Table 1 shows the leading seven growth rates, and the magnitude of the $\ell=1$ internal growth rates, for various numbers of patches, $m=4,8,16,32$. The exact growth rates of the diffusion PDE (16) are $\lambda=-k^{2}$ for integer $k$. See in the table that as the number of patches double, the accuracy of the growth rates of the macroscopic modes improves by a factor of about 16 . This is consistent with an $\mathcal{O}\left(H^{4}\right)$ method as predicted for diffusion with PtBC (17).

Second, we repeat the analysis for fewer subgrid points so that the microscopic dynamics are not resolved as well. Table 2 shows the leading eigenvalues for $n=7$ points in each patch. There is no significant difference between Tables 1 and 2 indicating that the microscopic resolution, the only difference between the two, has little impact on the macroscopic dynamics. No growth rate is significantly positive showing the numerical method is stable - the leading growth rate is close to zero corresponding to conservation of material. The other dominant growth rates rapidly approach those for diffusion.

Lastly, consider the diffusive dynamics of (16) when connected by the PtBC that at $x=X_{j} \pm r H$

$$
\begin{aligned}
H \partial_{x} v_{j}=[\mu \delta & \pm r \delta^{2}-\left(\frac{1}{6}-\frac{1}{2} r^{2}\right) \mu \delta^{3} \mp r\left(\frac{1}{12}-\frac{1}{6} r^{2}\right) \delta^{4} \\
& \left.+\left(\frac{1}{30}-\frac{1}{8} r^{2}+\frac{1}{24} r^{4}\right) \mu \delta^{5} \pm r\left(\frac{1}{90}-\frac{1}{36} r^{2}+\frac{1}{120} r^{4}\right) \delta^{6}\right] U_{j} .
\end{aligned}
$$

Obtain this PtBC from the first six terms of (8) or equivalently from PtBC (10) 
TABLE 3: Growth rates of perturbations from steady state $u=0$ as for Table 1 but with the sixth order PtBC (18).

\begin{tabular}{|r|lccl|c|}
\hline$m$ & 1 & 2,3 & 4,5 & 6,7 & $m+1: 2 m$ \\
\hline 4 & $8 \times 10^{-12}$ & -0.982238 & -2.457648 & n/a & -99.79 \\
8 & $4 \times 10^{-11}$ & -0.999677 & -3.928952 & -7.843254 & -399.1 \\
16 & $4 \times 10^{-11}$ & -1.000006 & -3.998708 & -8.967122 & -1596. \\
32 & $-2 \times 10^{-10}$ & -1.000003 & -4.000023 & -8.999625 & -6386. \\
\hline
\end{tabular}

by discarding $\mathcal{O}\left(\gamma^{4}\right)$ terms. Table 3 demonstrates that the resultant numerical scheme is stable and has sixth order consistency for the diffusion equation. Further, it is these PtBCs we used to simulate the nonlinear dynamics of Burgers' equation (1) to create Figures 1 and 2.

\section{Conclusion}

We achieve higher order accuracy in the gap-tooth scheme using carefully crafted patch boundary conditions (PtBCs). Analytic approximations and analysis of numerical steps in time confirm the PtBCs (17) and (18) are effective. Importantly, the PtBCs (17) and (18) do not depend upon the particular PDE being simulated, thus the PtBCs should work effectively for particle simulations for which we do not have an algebraic microscale closure.

Further, although the predicted microscopic subgrid scale fields do have some dependence upon the patch size $r$, the dependence weakens, by being pushed to higher orders in $r$, in using higher order accuracy patch boundary conditions.

As shown in Figures 1 and 2, the PtBCs we recommend here appear to work well even for the nonlinear dynamics of Burgers equation (1).

The research reported here is a crucial part of our project to establish 
some currently missing, crucial synergism between "conventional" numerical analysis on the one hand, and microscopic complex systems modeling on the other: bridging systematically the enormous gap between the microscopic description of a complex physical/material system and system-level simulation and analysis of direct systems/engineering importance.

Acknowledgment: I. G. K. is supported in part by DARPA and an NSF/ ITR grant.

\section{References}

[1] Vemuri Balakotaiah and Hsueh-Chia Chang. Hyperbolic homogenized models for thermal and solutal dispersion. SIAM J. Appl. Math., 63:1231-1258, 2003.

http://epubs.siam.org/sam-bin/dbq/article/36886. C639

[2] J. Carr. Applications of centre manifold theory, volume 35 of Applied Math. Sci. Springer-Verlag, 1981. C647

[3] J. Carr and R. G. Muncaster. The application of centre manifold theory to amplitude expansions. II. Infinite dimensional problems. J. Diff. Eqns, 50:280-288, 1983. C647

[4] J. Cisternas, C. W. Gear, S. Levin, and I. G. Kevrekidis. Equation-free modeling of evolving diseases: Coarse-grained computations with individual-based models. Technical report, [http://arXiv.org/abs/nlin.A0/0310011], 2003. C639, C641

[5] J. Dolbow, M. A. Khaleel, and J. Mitchell. Multiscale mathematics initiative: A roadmap. report from the 3rd DoE workshop on multiscale mathematics. Technical report, Department of Energy, USA, http://www.sc.doe.gov/ascr/mics/amr, December 2004. C639 
[6] C. W. Gear, I. G. Kevrekidis, and C. Theodoropoulos. 'Coarse' integration/bifurcation analysis via microscopic simulators: micro-galerkin methods. Computers and Chemical Engrg, 26:941-963, 2002. C643

[7] C. W. Gear, Ju Li, and I. G. Kevrekidis. The gap-tooth method in particle simulations. Phys. Lett. A, 316:190-195, 2003. C640, C641, C645

[8] Igor Jankovic, Aldo Fiori, and Gedeon Dagan. Effective conductivity of an isotropic heterogeneous medium of lognormal conductivity distribution. Multiscale Modeling and Simulation, 1:40-56, 2003. http://epubs.siam.org/sam-bin/dbq/article/40963. C639

[9] I. G. Kevrekidis, C. W. Gear, and G. Hummer. Equation-free: the computer-assisted analysis of complex, multiscale systems.

A. I. Ch. E. Journal, 50:1346-1354, 2004. C639, C641

[10] I. G. Kevrekidis, C. W. Gear, J. M. Hyman, P. G. Kevrekidis, O. Runborg, and K. Theodoropoulos. Equation-free, coarse-grained multiscale computation: enabling microscopic simulators to perform system level tasks. Comm. Math. Sciences, 1:715-762, 2003. C639

[11] J. Li, C. W. Gear P. G. Kevrekidis, and I. G. Kevrekidis. Deciding the nature of the coarse equation through microscopic simulation: an augmented Lagrangian approach. SIAM Multiscale Modeling and Simulation, 1:391-407, 2003. http://epubs.siam.org/sam-bin/dbq/article/41916. C641

[12] T. Mackenzie and A. J. Roberts. Holistic finite differences accurately model the dynamics of the Kuramoto-Sivashinsky equation. ANZIAM J., 42(E):C918-C935, 2000. http://anziamj.austms.org.au/V42/CTAC99/Mack. C644

[13] T. MacKenzie and A. J. Roberts. Holistic discretisation of shear dispersion in a two-dimensional channel. In K. Burrage and Roger B. 
Sidje, editors, Proc. of 10th Computational Techniques and Applications Conference CTAC-2001, volume 44, pages C512-C530, March 2003. http://anziamj. austms.org. au/V44/CTAC2001/Mack. C643

[14] G. W. Milton. Theory of Composites. Cambridge University Press, 2002. http://www. cambridge.org/uk/catalogue/catalogue. asp? isbn=0521781256. C639

[15] National Physical Laboratory. Modern Computing Methods, volume 16 of Notes on Applied Science. Her Majesty's Stationary Office, 1961. C644

[16] A. J. Roberts. Low-dimensional modelling of dynamics via computer algebra. Computer Phys. Comm., 100:215-230, 1997. C648

[17] A. J. Roberts. Holistic discretisation ensures fidelity to Burgers' equation. Applied Numerical Modelling, 37:371-396, 2001. C643, C644, C646

[18] A. J. Roberts. A holistic finite difference approach models linear dynamics consistently. Mathematics of Computation, 72:247-262, 2002. http://www. ams.org/mcom/2003-72-241/S0025-5718-02-01448-5. C643, C646

[19] A. J. Roberts. A step towards holistic discretisation of stochastic partial differential equations. In Jagoda Crawford and A. J. Roberts, editors, Proc. of 11th Computational Techniques and Applications Conference CTAC-2003, volume 45, pages C1-C15, December 2003. http://anziamj . austms . org.au/V45/CTAC2003/Robe/home.html [December 14, 2003]. C643

[20] A. J. Roberts. Analyse gap-tooth patch boundary conditions with Reduce. Technical report, http://www.sci.usq.edu.au/staff/aroberts/linpbc.red, September 2004. C648, C649 
[21] G. Samaey, I. G. Kevrekidis, and D. Roose. Damping factors for the gap-tooth scheme. In S. Attinger and P. Koumoutsakos, editors, Multiscale Modeling and Simulation, volume 39 of Lecture Notes in Computational Science and Engineering, pages 93-102. Springer-Verlag, 2004. C641, C651

[22] G. Samaey, I. G. Kevrekidis, and D. Roose. The gap-tooth scheme for homogenization problems. SIAM Multiscale Modeling and Simulation, to appear. C639, C640, C641

[23] Dongbin Xiu and Ioannis G. Kevrekidis. Equation-free, multiscale computation for unsteady random diffusion. Technical report, [http://arXiv.org/abs/math.NA/0504273], 2005. C640

[24] Xinye Yue Zhiming Chen. Numerical homogenization of well singularities in the flow transport through heterogeneous porous media. Multiscale Modeling and Simulation, 1:260-303, 2003. http://epubs.siam.org/sam-bin/dbq/article/41332. 